\title{
Polymorphism RS2227306 In The Interleukin 8 Gene and Its Relation to Bronchial Asthma: Review Article
} Sahbaa Fehr Mohamed Hafez ${ }^{1}$, Osama Taha Amer ${ }^{* 1}$, Osama Abd Elaziz Mostafa ${ }^{1}$, Walaa Mohamed Samy ${ }^{2}$ Departments of ${ }^{1}$ Pediatrics and ${ }^{2}$ Medical Biochemistry, Faculty of Medicine, Zagazig University, Egypt *Corresponding author: Osama Abd Elaziz Mostafa Abd Elaziz, Email: drosama.smsm@gmail.com

\begin{abstract} relation to bronchial asthma. of severity of asthma.

\section{INTRODUCTION}

Asthma is a life-threatening respiratory disorder. Persistent airway inflammation is the hallmark of this common lung illness. A chronic inflammatory disease of the airways, asthma, is characterised by symptoms like chronic wheezes and dyspnea, as well as tightness in the chest and coughing, especially in the early morning or late at night. These episodes are frequently accompanied by a wide-spread, yet variable, obstruction of airflow, which can be resolved either naturally or with treatment ${ }^{(\mathbf{1})}$.

Asthma frequency among youngsters globally has increased gradually during the past two decades. An estimated 8.2 percent of Egyptian youngsters aged 3 to 15 were asthmatic ${ }^{(2)}$.
\end{abstract}

Background: Allergy symptoms such as wheezing, breathlessness, and coughing are all symptoms of asthma, which is a long-term respiratory condition characterized by inflammation and reversible airway blockage. If you're a child or young adult, asthma is the most common long-term condition, with more severe symptoms. The CXC chemokine superfamily includes the Interleukin 8 (IL-8) chemokine. T lymphocytes, neutrophils, and mast cells are all chemotactic cytokines that IL-8 attracts in the body. A polymorphism in the promoter region of IL-8 - 781C/T (rs2227306) has been found to be associated with an elevated level of the cytokine IL-8. There is a gene in the first intron of the IL-8 - 781C/T (rs2227306) that has been reported to aid in both gene transcription and gene regulation.

Objective: The aim of the present review was to study polymorphism RS2227306 in the interleukin 8 gene and its

Conclusion: IL8 polymorphism rs2227306 has the potential to be utilized as a marker in interpretation of assessment

Keywords: Polymorphism Rs2227306, Interleukin 8, Bronchial asthma.

Objective: To find out if the IL-8 polymorphism is connected to asthma.

\section{Chronic inflammation and IL-8:}

Numerous cell types, as endothelial cells, macrophages, neutrophils, fibroblasts, and tumor cells produce IL-8. Several cell types produce it in reaction to inflammation ${ }^{(4)}$.

One of the most significant mediators of inflammation and wound healing, IL-8 acts as an activator of neutrophils, which in turn activates $\mathrm{T}$ and nonspecific inflammatory cells. In human fibroblasts, it also increases the synthesis of $\alpha$-smooth muscle actin. As a result, IL-8 promotes wound healing in vivo by attracting fibroblasts, promoting their migration and the deposition of collagen I, fibronectin, and tenascin ${ }^{(4,5)}$.

The IL8 gene consists of, 3 introns, 4 exons and the proximal promoter, which is found on chromosome $4 \mathrm{q} 13$ -
21. The IL8 gene has been found to have many polymorphisms ${ }^{(6)}$. The IL-8 production can also be regulated by certain of these organisms. Several single nucleotide polymorphisms (SNPs) in the IL8 gene, such as rs2227532, rs738T/C, rs4073, rs396T/G, and rs2227306, have been investigated in patients with aggressive $(\mathrm{AgP})$ periodontitis or chronic periodontitis (CP) among people of Brazil $^{(7)}$.

\section{IL-8's Inflammatory Properties:}

Epithelial cells emit IL-8, a proinflammatory chemokine that responds to oxidative stress and causes neutrophil infiltration and inflammation when exposed to an oxidising particle. IL-8 is a powerful chemoattractant and neutrophil activator ${ }^{(8)}$. Post-injury regulation of IL-8 depend on proinflammatory stimuli. IL-8 plays a critical role in a number of wound healing pathways. As a chemotactic agent for leukocytes, and fibroblasts, it also stimulates myofibroblast growth and angiogenesis ${ }^{(9)}$. When cells are subjected to stress, IL-8 is released, which is associated with an increase in proinflammatory cytokine production. By manipulating the expression levels of this cytokine in human cells, neutrophil infiltration into injured tissue can be controlled to a certain extent ${ }^{(\mathbf{8})}$.

\section{Lung Epithelial Cell Injury Prevention:}

Acute respiratory distress syndrome (ARDS) patients' BAL fluid contains high levels of TNF- $\alpha^{\left({ }^{(10)}\right.}$, TNF- and IL-8 levels are linked to each other. In lung epithelial cells, TNF- $\alpha$ is a key inducer of expression of IL-8 ${ }^{(11)}$. 


\section{Pathogenesis of asthma}

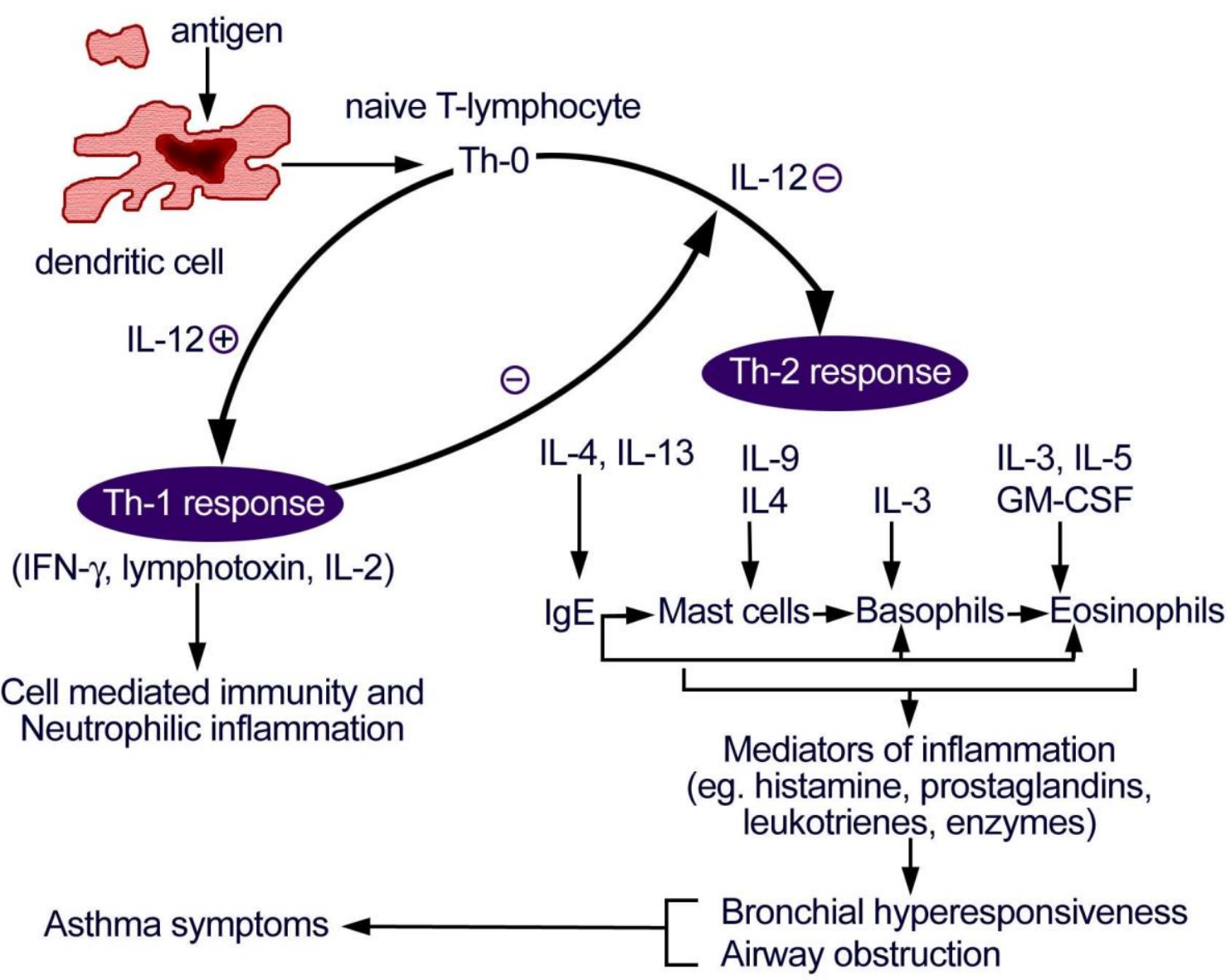

Figure (1): Pathogenesis of asthma ${ }^{(3)}$

\section{Pathogenesis of asthma (Figure 1): \\ Role in Asthma:}

Infancy IL-8 deficiency may be the root cause of bronchial asthma and severe respiratory syncytial virus (RSV) infections, wheeze and/or bronchial asthma may occur as a result ${ }^{(\mathbf{1 2})}$. Asthma patients had higher levels of IL-8 in their BAL fluid and sputum ${ }^{(13)}$. Guinea pigs' bronchial hyper-reactivity is also induced by repeated injections of IL-8 into the airways. Asthma and RSV bronchiolitis share a genetic link with IL-8 ${ }^{\left({ }^{(14)}\right.}$.

Highly specific for IL-8 are two distinct receptors: $\beta$ (IL-8RB, CXCR2), as well as $\alpha$ (IL-8RA, CXCR1). Members of the superfamily of receptors, which link to guanine nucleotide-binding proteins, are these closely-related proteins. An association between the IL-8RA gene and total serum IgE levels in asthmatic patients has been shown ${ }^{(15)}$.

These polymorphisms have been linked to a variety of respiratory conditions, including asthma and chronic obstructive pulmonary disease (COPD). Asthma and severe RSV infections aren't influenced by IL-8RA polymorphisms, according to the latest research ${ }^{(\mathbf{1 6})}$.

A correlation between IL-8 expression levels and the probability of developing asthma in a child:

There are two receptors for CXC chemokines in the IL-8 family: CXCR1 and CXCR2. CXCR1 is the IL-8 RA, and CXCR2 is the IL- 8 beta (IL-8 RB, CXCR2). Neutrophils, mononuclear phagocytosis, mast cells, and T-cells are all activated by the IL8-induced recruitment of inflammatory cells ${ }^{(\mathbf{1 7})}$.

Acute and chronic inflammation are both triggered by IL8. ARDS, severe respiratory syncytial virus infections, viral bronchiolitis, and bronchial asthma are all thought to be caused in part by this molecule's presence ${ }^{(\mathbf{1 6})}$. Asthma patients' neutrophils and airway epithelial cells are expanded as a result of the release of proinflammatory cytokines such as IL- 8 by Th2, Th-9, and Th-17 cells, according to several studies ${ }^{(\mathbf{1 8})}$.

Several studies have found a link between asthma severity and elevated neutrophil counts ${ }^{\left({ }^{(19)}\right.}$. IL-8 levels may have risen because of the link between IL- 8 and 
neutrophil recruitment following lung damage. Indeed, IL-8 and/or other chemokines are released into the airways by human bronchial epithelium, fibroblast, and smooth muscle cells. Biomarkers of illness progression and severity may include IL-8, which may play an important role in the development of asthmatic airway inflammation and hyper-responsiveness ${ }^{(\mathbf{1 8})}$.

In addition, two IL8 variations were examined for their connection with pediatric asthma susceptibility. The rs4073 and rs2227306 loci have been linked to an increased incidence of asthma in children. According to these findings, genetic variants in IL-8 may have a role in the development of pediatric asthma ${ }^{(\mathbf{1 9})}$.

There is a possibility that genetic polymorphisms have significant functional implications. These changes have the potential to alter the protein's expression or activity. The IL 8 transcriptional activity was found to be impacted by the two polymorphisms rs 4073 and rs2227306. Possible effects of IL-8 variants may include affecting another gene's expression, such as an SNP in a gene's promoter region, which may have no effect on the expression of IL-8 (mRNA or protein) alone. Second, the results were not as accurate as they could have been when SNPs were not examined inside the correct haplotype blocks (some SNPs in the same region are in LD with other SNPs) ${ }^{(\mathbf{1 7})}$.

$\mathrm{T}$ allele rs2227306 is associated with C/EBP-b binding preference in lung epithelial cells, according to Hacking and colleagues ${ }^{(20)}$. A key regulator of inflammation, C/EBP $b$, binds to a regulatory region in the IL8 gene, increasing transcription by a large amount (20).

In a study conducted by Heinzmann et al. ${ }^{(21)}$, IL-8 was tested for its association with asthma and arthritis, as well as to see if RSV bronchiolitis and asthma share a common genetic basis. Finding a link between asthma and all of a person's polymorphisms, it is concluded that IL-8 has a connection with asthma and that JIA has a strong inverse distribution of polymorphisms ${ }^{(\mathbf{2 1})}$.

\section{Interleukin 8 gene polymorphism Rs2227306:}

CXC chemokine, Interleukin 8 (OMIM 146930) has significant effects on peripheral blood neutrophil activation and tissue migration ${ }^{(22,23)}$.

Case-control studies have been used extensively to explore genetic variations in the IL8 gene, which is situated on chromosome 4q12-q21 and has numerous single nucleotide polymorphisms (SNP) that are associated with acute inflammation and chronic inflammation. +396(G/T), +781(T/C) and $-251(\mathrm{~A} / \mathrm{T})$, SNPs in the IL8 gene are among the most studied ${ }^{(23)}$. An intron-located polymorphism, rs2227306, is associated with gene transcription and regulation, while rs4073rs2227306 haplotype exerted the strongest influence on IL-8 expression ${ }^{(24)}$.

IL-8 expression is unaffected by other polymorphisms, although numerous studies have found a link between those polymorphisms and an increased risk of cancer. On the other hand, a study by Liu and colleagues ${ }^{(25)}$ found that four polymorphisms (rs4073, rs2227306, $+1633 \mathrm{C} / \mathrm{T}$ and $+276 \mathrm{~A} / \mathrm{T}$ ) were not associated with pathological characteristics or oral cancer risk.

It has been extensively reported that IL-8 polymorphisms are associated with an increased risk of cancer, although the findings have been contested. In a meta-analysis and comprehensive review, Zhang et al. ${ }^{\text {(24) }}$ investigated the relationship between IL-8 polymorphisms (+1633C/T, rs2227306, rs2227307, rs1126647, and $+678 \mathrm{~T} / \mathrm{C}$ ) and cancer risk. They concluded that the rs2227306 polymorphism protects against hepatocellular carcinoma risk based on the results of their study. To confirm their findings, future welldesigned research with a bigger sample size are necessary.

Variants like rs2227306 (+781C/T; rs2227306) have been discovered to be related to the transcriptional level of IL-8, suggesting that they may be involved in the onset or progression of many disorders (26): 1- Excessive production of an IL-8 gene that has been linked to an increased risk of respiratory syncytial virus disease ${ }^{(\mathbf{2 0})}, \mathbf{2}$ Increased incidence of wetage-related macular degeneration in Taiwan Chinese without the CFH Y402H polymorphism but with the IL- $8+781$ T allele (AMD) ${ }^{(25)}$ and 3-Polymorphism in the $+781 \mathrm{C} / \mathrm{T}$ allele of the IL-8 gene investigated in this study reveals its prospective role as an inflammatory marker for both chronic kidney disease (CKD) and COPD ${ }^{(25)}$.

\section{CONCLUSION}

There has been substantial research into the use of IL8 polymorphism rs2227306 occurrence for interpretation of asthma severity.

\section{Financial support and sponsorship: Nil.} Conflict of interest: Nil.

\section{REFERENCES}

1. Zubair M, Kumar M, Khan M et al. (2018): Asthma: an overview. European Journal of Biomedical, 5 (5): 919-924.

2. Hassan A, Hagrass S (2017): Pediatric Department, Al-Azhar University, Assiut, Egypt and Community Health, Nursing Department, Faculty of Nursing, Zagazig University, Zagazig, Egypt. Prevalence of bronchial asthma in primary school children. Am J Med Med Sci., 7: 67-73.

3. Morris M (2016): Asthma treatment and management. Available http://emedicine.medscape.com/article/296301treatment\#aw2aab6b6b7.

4. Gonzalez-Aparicio M, Alfaro C (2019): Significance of the IL-8 pathway for immunotherapy. Human Vaccines \& Immunotherapeutics, 18: 1-6.

5. Henry G, Garner, W L (2003): Inflammatory mediators in wound healing. The Surgical clinics of North America, 83 (3): 483-507.

6. Rojas M, Rodríguez Y, Leon $\mathrm{K}$ et al. (2018): Cytokines and inflammatory mediators in systemic lupus erythematosus. Rheumatology, 11: 1-5. 
7. Andia D, Letra A, Casarin R et al. (2013): Genetic analysis of the IL8 gene polymorphism (rs4073) in generalized aggressive periodontitis. Archives of Oral Biology, 58 (2): 211-217.

8. Qazi B, Tang K, Qazi A (2011): Recent advances in underlying pathologies provide insight into interleukin8 expression-mediated inflammation and angiogenesis. International Journal of Inflammation, 11: 132-138.

9. Beserra F, Gushiken L, Hussni M et al. (2018): Regulatory Mechanisms and Chemical Signaling of Mediators Involved in the Inflammatory Phase of Cutaneous Wound Healing. In Wound Healing-Current Perspectives. Intech Open, 18: 122-127.

10. Suter U, Welcher A, Özcelik T et al. (1992): Trembler mouse carries a point mutation in a myelin gene. Nature, 356 (6366): 241-244.

11. Stoehr L, Endes C, Radauer-Preiml I et al. (2015): Assessment of a panel of interleukin-8 reporter lung epithelial cell lines to monitor the pro-inflammatory response following zinc oxide nanoparticle exposure under different cell culture conditions. Particle and Fibre Toxicology, 12 (1): 29-35.

12. Vázquez Y, González L, Noguera L et al. (2019): Cytokines in the respiratory airway as biomarkers of severity and prognosis for Respiratory Syncytial Virus infection: an update. Frontiers in Immunology, 10: 1-4.

13. Hosoki K, Ying S, Corrigan C et al. (2015): Analysis of a panel of 48 cytokines in BAL fluids specifically identifies IL-8 levels as the only cytokine that distinguishes controlled asthma from uncontrolled asthma, and correlates inversely with FEV1. PloS One, 10 (5): $1-5$.

14. Xiu Q, Fujimura M, Nomura M et al. (1995): Bronchial hyperresponsiveness and airway neutrophil accumulation induced by interleukin- 8 and the effect of the thromboxane A2 antagonist S-1452 in guineapigs. Clinical \& Experimental Allergy, 25 (1): 51-59

15. Jiang N, Yan Z, Wang J et al. (2016): Oxidative stress and endocytosis are involved in upregulation of interleukin-8 expression in airway cells exposed to PM 2.5. Environmental Toxicology, 31 (12): 1869-1878.

16. Pinto L, DE AzeredoLeita $\sim$ o $\mathrm{L}$, Mocellin $M$ et al. (2017): IL-8/IL-17 gene variations and the susceptibility to severe viral bronchiolitis. Epidemiol Infect., 145 (4): 642-646.

17. Charrad R, Kaabachi W, Rafrafi A et al. (2017): IL8 gene variants and expression in childhood asthma. Lung, 195 (6): 749-757.

18. Vock C, Hauber H, Wegmann $M$ (2010): The other $T$ helper cells in asthma pathogenesis. Journal of Allergy, 154: 1-5.

19. Lindén A (2001): Role of interleukin-17 and the neutrophil in asthma. International Archives of Allergy and Immunology, 126 (3): 179-184.

20. Hacking D, Knight J, Rockett $\mathrm{K}$ et al. (2004): Increased in vivo transcription of an IL-8 haplotype associated with Respiratory syncytialvirus diseasesusceptibility. Genes Immun., 5 (4): 274-28.

21. Heinzmann A, Ahlert I, Kurz T et al. (2004): Association study suggests opposite effects of polymorphisms within IL8 on bronchial asthma and respiratory syncytial virus bronchiolitis. J Allergy ClinImmunol., 114 (3): 671-676.

22. Viana A, de Carvalho M, Kim Y et al. (2009): 〈b $>$ Amplification-Created Restriction Site (Acrs) Method For The Detection Of The Polymorphism Rs2227306 In The Interleukin 8 Gene And Interethnic Comparison of Genotype Distribution. Saúde e Pesquisa, 2 (1): 27-31.

23. Zhang M, Fang T, Wang K et al. (2016): Association of polymorphisms in interleukin-8 gene with cancer risk: a meta-analysis of 22 case-control studies. OncoTargets and Therapy, 9: 3727-32.

24. Shanmuganathan $\mathbf{R}$, Ramanathan $K$, Padmanabhan G et al. (2017): Evaluation of Interleukin 8 gene polymorphism for predicting inflammation in Indian chronic kidney disease and peritoneal dialysis patients. Alexandria Journal of Medicine, 53 (3): 215-220.

25. Liu C, Yeh C, Yu C et al. (2012): Impact of interleukin-8 gene polymorphisms and environmental factors on oral cancer susceptibility in Taiwan. Oral Dis., 18: 307-314.

26. Tsai Y, Lin J, Wan L et al. (2008): Interleukin gene polymorphisms in age- related macular degeneration. Invest Ophthalmol Vis Sci., 49: 693-698. 